The Astrophysical Journal, 178:125-130, 1972 November 15

(C) 1972. The American Astronomical Society. All rights reserved. Printed in U.S.A.

\title{
MOLECULAR CLOUDS IN THE GALACTIC CENTER REGION: CARBON MONOXIDE OBSERVATIONS AT 2.6 MILLIMETERS
}

\author{
P. M. Solomon \\ School of Physics and Astronomy, University of Minnesota \\ N. Z. SCOVILLE* \\ Astronomy Department, Columbia University \\ AND \\ K. B. Jefferts, $\uparrow$ A. A. Penzias, $\ddagger$ and R. W. Wilson $\dagger$ \\ Bell Laboratories \\ Received 1972 April 26; revised 1972 June 12
}

ABSTRACT

A preliminary $\mathrm{CO}$ emission line survey covering a strip at $b=-2^{\prime}$ from $l=359.7$ to $l=2.8$ is presented which shows a continuous band of emission connecting the region between $\mathrm{Sgr} \mathrm{A}$ and Sgr B. A high-resolution map of the Sgr A cloud near the galactic center shows that there are at least two clouds centered within $3^{\prime}$ of each other with a velocity difference of $35 \mathrm{~km} \mathrm{~s}^{-1}$. Measurement of the ${ }^{13} \mathrm{C}^{16} \mathrm{O}$ and ${ }^{12} \mathrm{C}^{18} \mathrm{O}$ emission indicates isotopic abundances similar to those of the solar system.

\section{INTRODUCTION AND OBSERVING PROCEDURE}

Microwave transitions from more than 20 molecules have been observed in the molecular clouds near the galactic-center radio sources Sgr A and Sgr B. Among all the transitions observed to date the ${ }^{12} \mathrm{CO}$ and ${ }^{13} \mathrm{CO} J=1-0$ millimeter emission lines at $115,271.2$ and $110,201.4 \mathrm{MHz}$ are the most useful as a probe of overall galactic structure as well as the kinematics and physical conditions of dense interstellar clouds. Observations of emission lines near H II regions (Penzias, Jefferts, and Wilson 1971) and in dark interstellar clouds (Penzias et al. 1972; Solomon 1972) show that carbon monoxide is more abundant than other interstellar molecules excepting $\mathrm{H}_{2}$, by a factor of $10^{3}-10^{5}$.

An important characteristic of the $\mathrm{CO}$ transition which differentiates it from other observed $\mathrm{mm}$ transitions is the long lifetime of the upper $J=1$ state, $A^{-1}=1.6 \times$ $10^{7} \mathrm{~s}$. This transition can therefore be thermalized by collisions at a hydrogen (atoms and/or molecules) density $N>A k T / \sigma v h v \sim 10^{3} \mathrm{~cm}^{-3}$. Carbon monoxide emission above the cosmic microwave background can therefore be expected from all regions with $N>10^{3} \mathrm{~cm}^{-3}$. This corresponds approximately to all clouds with greater than 1 mag extinction per parsec. Unlike the $18-\mathrm{cm} \mathrm{OH}$ and 6- $\mathrm{cm} \mathrm{H}_{2} \mathrm{CO}$ lines, which have been used for mapping, the $2.6-\mathrm{mm}$ CO line is observed only in emission and at a wavelength where the continuum radiation from the thermal and nonthermal galactic sources can be ignored. Carbon monoxide observations thus offer a direct view of all dense regions in the Galaxy regardless of their position relative to $\mathrm{H}$ II regions and nonthermal sources.

In this paper we present the results of a preliminary survey of ${ }^{12} \mathrm{CO}$ emission in the galactic center covering a strip in the plane of the Galaxy at $b=-2^{\prime}$ from $l=359.7$

* Present address: School of Physics and Astronomy, University of Minnesota.

$\dagger$ Murray Hill, New Jersey.

$\ddagger$ Holmdel, New Jersey. 
to $l=2: 8$, with observations spaced every $6^{\prime}$ (at $l>2: 0$ the observations were $12^{\prime}$ apart), and a map of the cloud near Sgr A with $1^{\prime}$ spatial resolution in the central regions and $2^{\prime}$ in the outer portions. In addition, about a dozen observations of the ${ }^{13} \mathrm{CO}$ lines were made in this region.

Observations were carried out with the $\mathrm{NRAO}^{1} 36$-foot antenna which has a beam efficiency of $\sim 60$ percent at $2.6 \mathrm{~mm}$ and a beamwidth of $\sim 1^{\prime}$. A 40-channel line receiver was used with a bandwidth per channel of $\sim 1.8 \mathrm{MHz}$ and a separation of $1 \mathrm{MHz}$. (1 MHz $=2.6 \mathrm{~km} \mathrm{~s}^{-1}$ for the ${ }^{12} \mathrm{CO}$ line.) Frequency switching was employed in all observations with the reference $58 \mathrm{MHz}$ below the signal frequency, i.e., 150 $\mathrm{km} \mathrm{s}^{-1}$ higher in velocity. The galactic-plane survey covered the velocity range $28 \leq V_{\mathrm{LSR}} \leq 130$, and the map of the $\mathrm{Sgr}$ A cloud was obtained for $-2 \leq V_{\mathrm{LSR}}$ $\leq 101$. Integrations were either 5 or 10 minutes with every third observation at an OFF position located about $2^{\circ}$ out of the galactic plane. The final data were obtained by subtracting adjacent on and oFF observations.

Calibrations were made by synchronously placing an absorbing sheet over the feed horn. Because atmospheric radiation acts to reduce the amplitude of the calibrating signal, the derived antenna temperatures are directly referred outside the atmosphere. Furthermore, it is an inherently frequency-independent calibration technique. Our very early work, in particular the previous CO observations (Penzias et al. 1971), made use of a rather sharply tuned noise diode, which was much less satisfactory. We estimate the 99 percent confidence limit of our present calibration error to be \pm 10 percent. In addition, our estimate of the beam efficiency of the antenna has a comparable uncertainty. Thus when uncertainties due to atmospheric absorption and scattering as well as noise in the records are included, it seems reasonable to assign an overall error limit of \pm 20 percent to the determination of the antenna temperature of the lines.

\section{GALACTIC-PLANE SURVEY}

The results of the galactic plane survey are presented in figure 1 in the form of antenna-temperature contours in the velocity-longitude plane. The effective velocity resolution is about $5 \mathrm{~km} \mathrm{~s}^{-1}$. Observations were made at $6^{\prime}$ intervals with our $\sim 1^{\prime}$ antenna beam, and the intermediate points on the map were obtained by interpolation. The 6' spacing was chosen to compare with the formaldehyde 6-cm survey of Scoville, Solomon, and Thaddeus (1972). (All formaldehyde data referred to are contained in that paper. The $\mathrm{H}_{2} \mathrm{CO}$ observations, however, included negative velocities not covered in this survey.)

The most outstanding characteristic of figure 1 is that $\mathrm{CO}$ emission with $T_{A}>2^{\circ} \mathrm{K}$ is observed over almost the complete velocity range between $l=359.8$ and 1.8 but is definitely weaker or absent between 1.8 and $2: 8$ at most velocities. All $\mathrm{H}_{2} \mathrm{CO}$ features (see Scoville et al., figs. $3 a$ and 5 and tables 1 and 2), including the four dominant $\mathrm{H}_{2} \mathrm{CO}$ clouds $5,10,13$, and 15 , are observed in $\mathrm{CO}$ at $l=0.0,0.7,1.1$, and 1.7, respectively. The $\mathrm{CO}$ line widths are all greater than $\mathrm{H}_{2} \mathrm{CO}$; this effect is undoubtedly due to the high optical depth in the $\mathrm{CO}$ line. However, all $\mathrm{CO}$ features do not have $\mathrm{H}_{2} \mathrm{CO}$ counterparts. In particular, the high-velocity $\mathrm{CO}$ features (see fig. 1) near Cloud $5(l=0.0)$ at $95>v>60$, near Cloud $10(l=0.7)$ at $105>v>80$, and connecting Cloud 5 and 10 from $v=60$ to 90 and $l=0.0-0.7$, are extremely weak or missing from the $\mathrm{H}_{2} \mathrm{CO}$ map. This is the first evidence of a continuous molecular region joining the two clouds near Sgr A and B (Cloud 5 and Cloud 10) which are separated by $0.7 \simeq 120 \mathrm{pc}$, at the distance of the galactic center. The most probable explanation for the difference in the $\mathrm{H}_{2} \mathrm{CO}$ absorption optical-depth maps and figure 1 is that the

\footnotetext{
${ }^{1}$ The National Radio Astronomy Observatory is operated by Associated Universities, Inc., under contract with the National Science Foundation.
} 


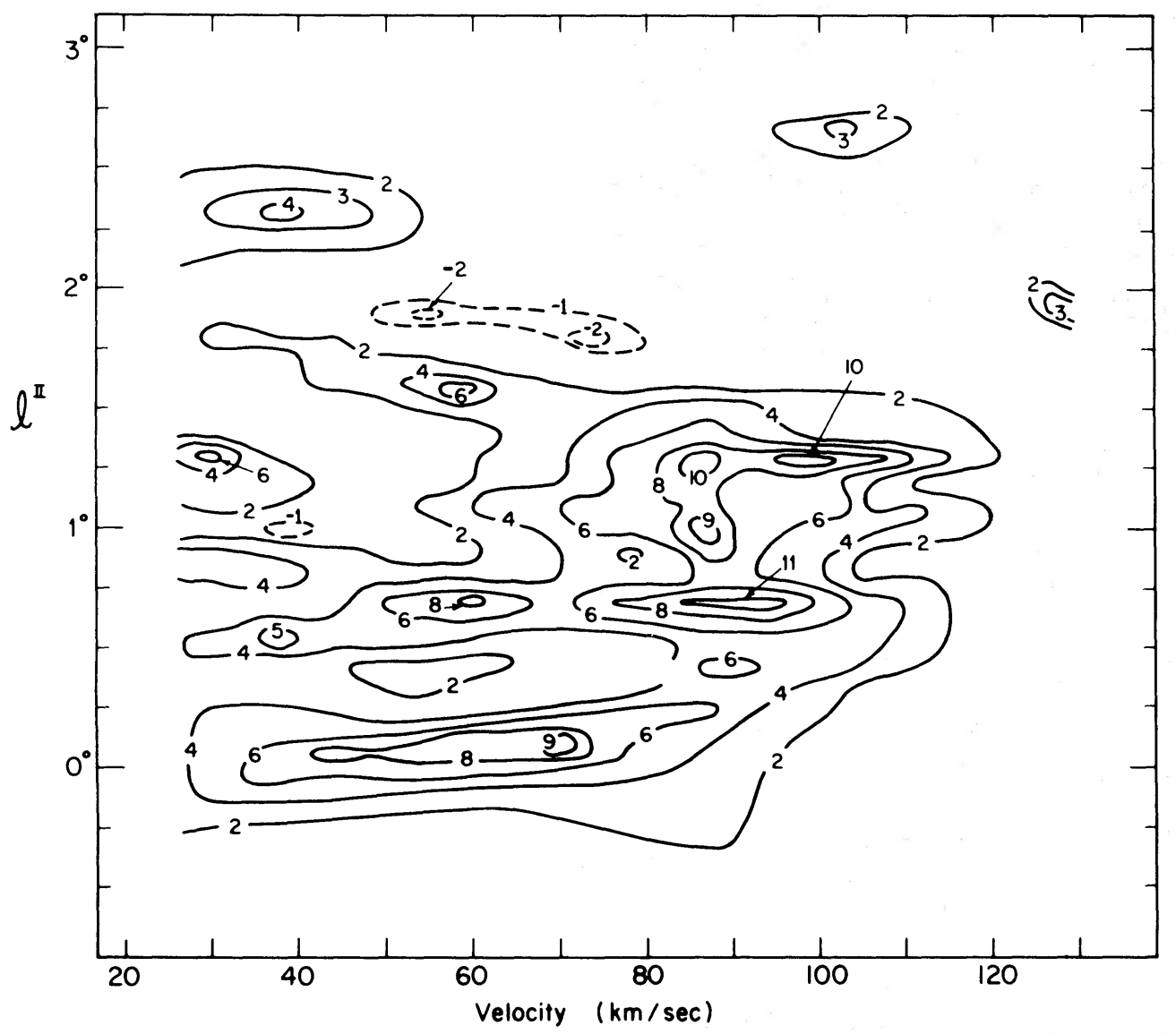

FIG. 1.-Contour diagram of ${ }^{12} \mathrm{CO}$ emission in the longitude-velocity plane at $b=-2^{\prime}$ from $l=359.7$ to $l=2: 8$. Contour unit at $1^{\circ} \mathrm{K} T_{A}\left(\approx 1.6^{\circ} \mathrm{K} T_{B}\right)$. Dashed contours are negative and represent possible emission at the reference velocity $v+150 \mathrm{~km} \mathrm{~s}^{-1}$.

clouds absent in $\mathrm{H}_{2} \mathrm{CO}$ are behind the source of the 6-cm continuum leading to a severe underestimate of the $\mathrm{H}_{2} \mathrm{CO}$ absorption optical depth. An interpretation of the kinematics of the molecular clouds in the galactic nuclear region in terms of an expanding ring is given by Scoville (1972).

The typical peak antenna temperature at a given position is $T_{A}=8^{\circ}-10^{\circ} \mathrm{K}$. Allowing for beam efficiency of 60 percent, the maximum brightness temperature is $T_{B}=1.7 T_{A}=13^{\circ}-17^{\circ} \mathrm{K}$. The $\mathrm{CO}$ brightness temperature in the center of these optically thick lines may be expected to equal the kinetic temperature at a layer where $\tau=1$ (see Solomon 1972; Penzias et al. 1972). It is entirely possible, as is indicated by observations of excited-state molecules (e.g., $\mathrm{CH}_{3} \mathrm{CN}$ observations, see Solomon et al. 1971), that there are small central hot cores in these regions with much higher kinetic temperatures. The peak $100-\mu$ brightness temperature in this region has been measured by Hoffman, Frederick, and Emery (1971) to be in the range $17^{\circ}-20^{\circ} \mathrm{K}$. The two surveys approximately agree in the position of peak brightness, although the relative intensity compared to other clouds of the $l=1.1$ cloud $\left(\mathrm{H}_{2} \mathrm{CO}\right.$ Cloud $13,100-\mu$ source D) is higher in CO emission than in the $100-\mu$ survey. The approximate agreement between the kinetic temperature deduced from the $\mathrm{CO}$ observations and the $100-\mu$ peak brightness temperature can be readily explained if the gas and dust are in thermal equilibrium and the central region of each cloud is optically thick at $100 \mu$. This would require more than 200 mag of extinction at optical wavelengths. Since the 
opacity in the center of the CO line is undoubtedly much greater than that at $100 \mu$, the brightness temperature of the latter can be representative of the kinetic temperature of a deeper, perhaps hotter layer.

A group of observations perpendicular to the plane was carried out at $l=1.4$, and these showed the extent of the emission to be beyond $+14^{\mathrm{m}}$ and $-6^{\mathrm{m}}$ in latitude.

\section{CARBON MONOXIDE MAP OF CLOUD 5 (Sgr A Region)}

The results of the ${ }^{12} \mathrm{CO}$ observations are presented in figures $2 a-f$ in the form of a series of antenna-temperature contours in the (velocity, right ascension) plane with the declination varying from $+3^{\prime} 30^{\prime \prime}$ (fig. $2 a$ ) to $-6^{\prime} 30^{\prime \prime}$ (fig. $2 f$ ). All coordinates are displacements from the Sgr A 6-cm continuum peak $\alpha_{1950}=17^{\mathrm{h}} 42^{\mathrm{m}} 28^{\mathrm{s}}, \delta_{1950}=$ $-28^{\circ} 50^{\prime} 30^{\prime \prime}$. This four-dimensional map clearly shows two separate major features. The northern feature [Cloud $5(+55)$ ], visible in figures $2 a, 2 b$, and $2 c$, is centered at $v \sim 55 \mathrm{~km} \mathrm{~s}^{-1}$; and the southern cloud [Cloud 5(+20)] is centered at $v \sim 20 \mathrm{~km} \mathrm{~s}^{-1}$ and dominates figures $2 d, 2 e$, and $2 f$. The velocity variation of the peak emission $\left(+60 \mathrm{~km} \mathrm{~s}^{-1}\right.$ at $\Delta \delta={ }^{\circ} 3^{\prime} 30^{\prime \prime}$ to $+15 \mathrm{~km} \mathrm{~s}^{-1}$ at $\left.\Delta \delta=-6^{\prime} 30^{\prime \prime}\right)$ is definitely not linear across the cloud since the velocity gradient $d v / d \theta<3 \mathrm{~km} \mathrm{~s}^{-1}$ arc $\min ^{-1}$ at $-0^{\prime} 30^{\prime \prime}<$ $\delta<3^{\prime} 30^{\prime \prime}$ and $-6^{\prime} 30^{\prime \prime}<\delta<-2^{\prime} 30^{\prime \prime}$, but between $\delta=-0^{\prime} 30^{\prime \prime}$ and $-2^{\prime} 30^{\prime \prime}$ increases to $d v / d \theta \approx 15 \mathrm{~km} \mathrm{~s}^{-1}$ arc min ${ }^{-1}$. Actually an indication of both features at the same declination can be seen in figure $2 c$ where the low-velocity emission is separated by only $8^{\mathrm{s}}(\sim 1.8)$ from the high-velocity cloud. Spatial $(\Delta \alpha, \Delta \delta)$ maps of peak antenna temperature in Cloud $5(+55)$ and a similar map for Cloud $5(+20)$ are presented in figures $3 a$ and $3 b$.

The ${ }^{12} \mathrm{CO}$ features are heavily saturated, and in any interpretation of the intensity contours on these maps we must attempt to separate out the effects of the excitation temperature $T_{01}$ and optical depth $\tau$ on the antenna temperature which are related approximately by the transfer equation,

$$
T_{A}=0.6 T_{01}\left(1-e^{-\tau}\right) .
$$

To determine optical depth and isotopic abundances, about a dozen observations were made of the ${ }^{13} \mathrm{CO}\left(\mathrm{O} \equiv{ }^{16} \mathrm{O}\right)$ line at selected positions. Two of these line profiles are presented in figures $4 a$ and $4 b$ along with the ${ }^{12} \mathrm{CO}$ lines and the intensity ratio $T^{12} \mathrm{Co} / T_{13} \mathrm{co}$. Throughout the cloud $T^{{ }^{12} \mathrm{CO}} / T^{13} \mathrm{Co}$ typically has a minimum value between 4 and 8 and is a strong function of velocity. The intensity ratio is observed to increase in the line wings to a value of 25 before noise in the ${ }^{13} \mathrm{CO}$ spectrum dominates. Thus, 25 is a minimum value for the isotopic ratio ${ }^{12} \mathrm{C} /{ }^{13} \mathrm{C}$. Assuming a true isotope ratio equal to the solar-terrestrial value of 89 (see $\S$ IV), we may estimate the optical depth as a function of velocity from $\tau^{12} \mathrm{co}=89 T^{13} \mathrm{co} / T^{12} \mathrm{co}$, for all $T^{12} \mathrm{co} / T^{13} \mathrm{co} \gg 1$.

Figure $4 b$ shows a minimum $\tau \sim 4.7$ at $v \sim 40 \mathrm{~km} \mathrm{~s}^{-1}$ and two maxima of 11.3 and 12.6 at $v \sim 60$ and $v \sim 20$, demonstrating the existence of two separate optically thick clouds at the same position. This effect is not at all visible in the ${ }^{12} \mathrm{CO}$ antenna temperature which is instead representative of the excitation temperature since $\tau \gg 1$. The double line appears to rule out rigid-body rotation which has been suggested on the basis of $\mathrm{OH}$ observations (Kerr and Sandquist 1968) as an explanation of the velocity gradient. Figure $4 a$ shows observations at a position where both features overlap producing a high optical depth from $v=20$ to $v=65 \mathrm{~km} \mathrm{~s}^{-1}$.

The decrease in intensity of ${ }^{12} \mathrm{CO}$ emission at velocities where the optical depth $\tau>1$ can be best explained as a decrease in excitation temperature. An alternative model consisting of a large number of very small extremely dense clouds with a velocity distribution similar to the ${ }^{12} \mathrm{CO}$ line profile, frequently referred to as clumpiness, is inconsistent with the much narrower line width in ${ }^{13} \mathrm{CO}$. 


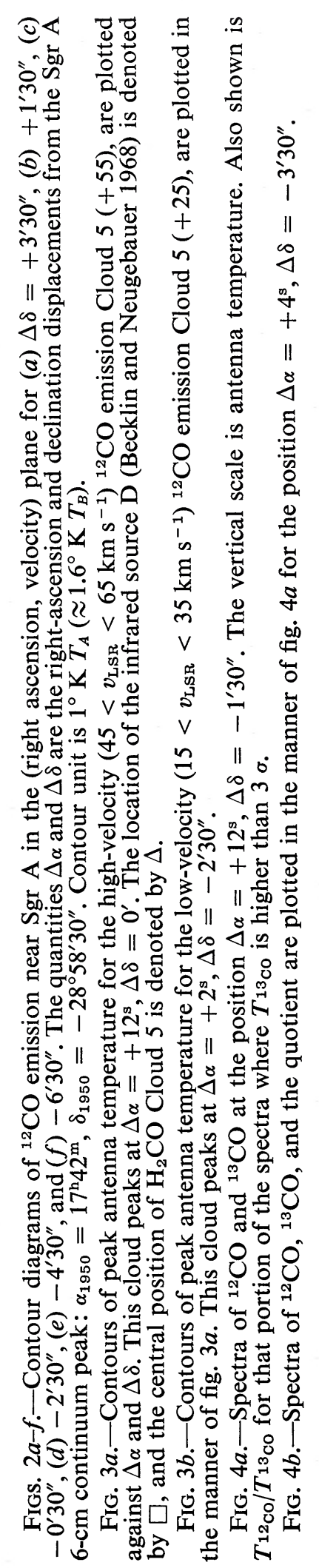



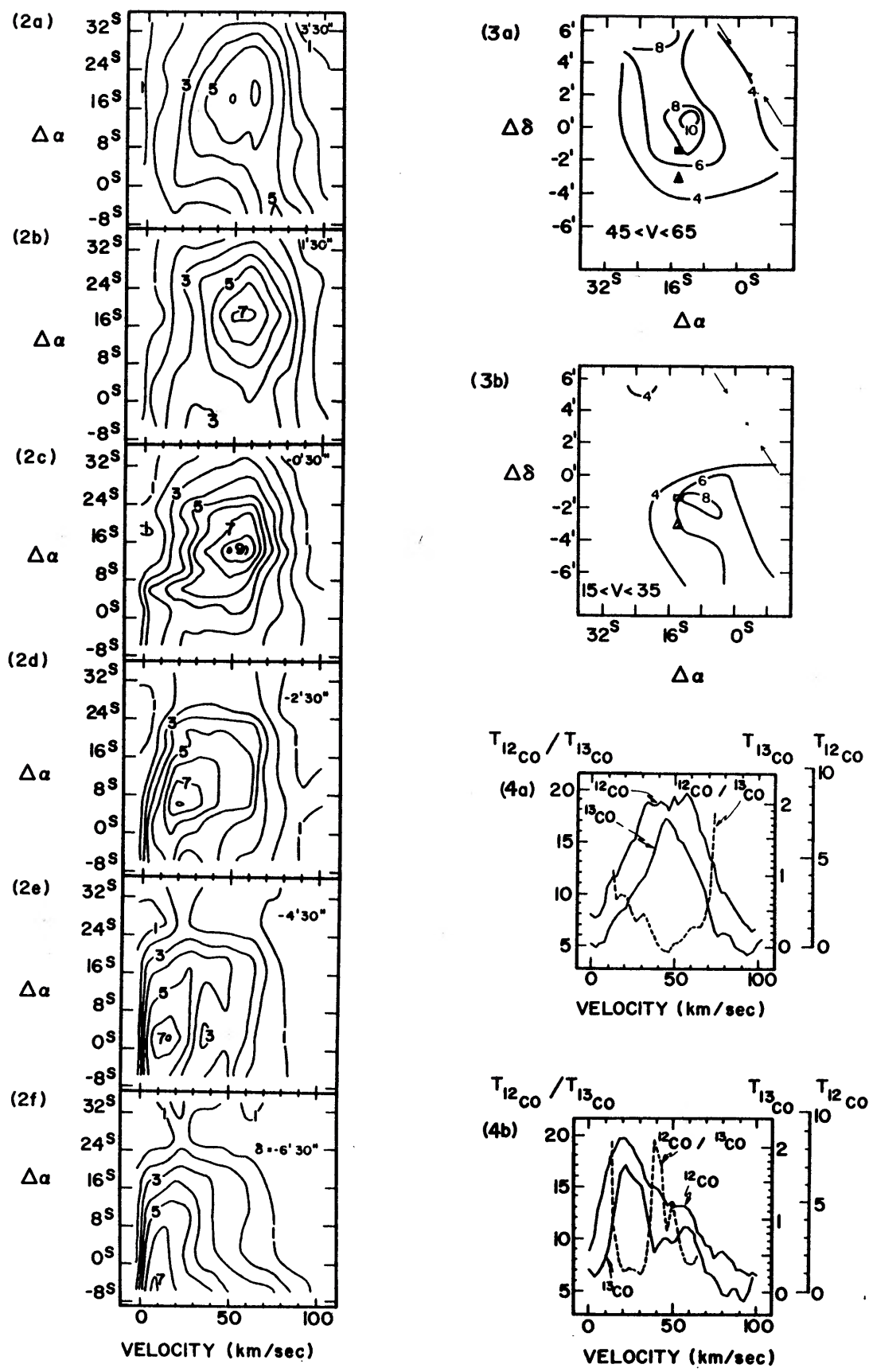
An estimate of the column density of $\mathrm{CO}$ can be obtained by assuming that the population of excited states is determined by a Boltzmann distribution at the observed brightness temperature $\left(T_{\mathrm{ex}}=T_{B}\right)$, which gives

$$
N_{\mathrm{Co}}=2.9 \times 10^{14} \int \frac{\tau(v) T_{B}(v)}{1-\exp \left[-5.5 / T_{B}(v)\right]} d v \mathrm{~cm}^{-2},
$$

where $\tau(v)$ must be determined from isotope measurements and the integration is over velocity in $\mathrm{km} \mathrm{s}^{-1}$. Determining $\tau(v)$ from the available ${ }^{13} \mathrm{CO}$ data we find typical column densities $N_{\mathrm{CO}} \sim 4-12 \times 10^{18} \mathrm{~cm}^{-2}$ within each of the two clouds. A lower limit to the total hydrogen column density, $N_{\mathrm{H}+2 \mathrm{H}_{2}}>10^{22} \mathrm{~cm}^{-2}$, is obtained by assuming all the carbon is in $\mathrm{CO}$ and an abundance ratio $\mathrm{C} / \mathrm{H}=3 \times 10^{-4}$. The angular sizes of the two clouds are difficult to determine because of the low ${ }^{13} \mathrm{CO}$ intensity expected near the cloud boundaries. However, their diameters are in the range $6^{\prime}-20^{\prime}$; and upon integrating the above hydrogen column density over the observed extent, we arrive at a firm lower limit for the mass of each cloud $\sim 10^{5} M_{\odot}$. This estimate is consistent with those obtained from $\mathrm{H}_{2} \mathrm{CO}$ observations. The position of maximum emission from these clouds definitely does not coincide with the Sgr A continuum peak as can be seen in figure 3 . The only feature approximately coinciding with the region is infrared source D from the map of Becklin and Neugebauer (1968).

\section{ISOTOPIC ABUNDANCES}

As part of our most recent observing program in 1972 April, we have detected the ${ }^{12} \mathrm{C}^{18} \mathrm{O}$ line at $109,782.2 \mathrm{MHz}$, during a $2 \frac{1}{2}$-hour integration at the position corresponding to figure $4 a$, in Cloud 5 . The ${ }^{12} \mathrm{C}^{18} \mathrm{O}$ emission has the same shape and central velocity as the ${ }^{13} \mathrm{C}^{16} \mathrm{O}$ line with a peak antenna temperature $T^{1{ }^{2} \mathrm{C}^{18} \mathrm{O}}=0.26$ $\pm 0.05^{\circ} \mathrm{K}$.

In the optically thin limit this yields an abundance ratio,

$$
\left({ }^{13} \mathrm{C}^{16} \mathrm{O}\right) /\left({ }^{12} \mathrm{C}^{18} \mathrm{O}\right)=1.004 T^{13} \mathrm{C}^{16} \mathrm{O} / T^{12} \mathrm{C}^{18} \mathrm{O}=6.6(+1.7,-1.0) .
$$

The optical depth of the isotopic lines can be determined, assuming that the excitation temperature is the same for all isotopic species including ${ }^{12} \mathrm{C}^{16} \mathrm{O}$, by using equation (1) which gives $\tau^{13} \mathrm{C}^{16} \mathrm{O}=0.26$; and $\tau^{12} \mathrm{C}^{18} \mathrm{O}=0.035 \pm 0.07$, yielding $\left({ }^{13} \mathrm{C}^{16} \mathrm{O}\right) /\left({ }^{12} \mathrm{C}^{18} \mathrm{O}\right)=$ $7.4(+1.9,-0.1)$. The difference between these two cases is not significant in view of the probable errors.

These observational isotopic ratios for two lines with small optical depth are in excellent agreement with the solar system value of $\left({ }^{13} \mathrm{C}^{16} \mathrm{O}\right) /\left({ }^{12} \mathrm{C}^{18} \mathrm{O}\right)=499 / 89=5.5$. The approximately 25 percent difference between the observed ratio in the galactic center and the solar-system ratio is well within the expected observational error. It thus appears that both the $\left({ }^{12} \mathrm{C} /{ }^{13} \mathrm{C}\right)$ and $\left({ }^{16} \mathrm{O} /{ }^{18} \mathrm{O}\right)$ abundance ratios in molecular clouds near the center of the Galaxy are similar to the solar-system values of 89 and 499 . While it is possible that the overabundance of one isotope and underabundance of the other could combine to produce this effect, we regard this chance coincidence as highly unlikely.

\section{CONCLUSIONS}

The principal results of this preliminary survey follow.

1. Carbon monoxide observations provide further evidence that extremely massive clouds, with $M>10^{5} M_{\odot}$, are concentrated in the galactic nuclear region. In addition to detecting all previously observed features, we have found a continuous band of emission connecting the region near Sgr A, at $l=0.0$ (Cloud 5) to the Sgr B (Cloud 10) region at $l=0.7$. 
2. The brightness temperature of the CO emission is close to the $100-\mu$ brightness temperature, suggesting that the grains and gas are at a similar temperature and that there may well be more than 200 mag of visual extinction in some of these regions.

3. A high-resolution map of Cloud 5 shows that there are actually at least two separate clouds centered within $3^{\prime}$ of each other with a velocity difference of $35 \mathrm{~km} \mathrm{~s}^{-1}$. Cloud 5 does not appear to be different from any of the other massive clouds within $200 \mathrm{pc}$ of the galactic center, although the apparent position of peak $\mathrm{CO}$ emission is only $3^{\prime}$ from Sgr A.

4. The isotopic abundances of carbon and oxygen appear to be approximately the same in the galactic center region as in the solar system.

\section{REFERENCES}

Becklin, E. E., and Neugebauer, G. 1968, Ap. J., 151, 145.

Hoffmann, W. F., Frederick, C. L., and Emery, R. J. 1971, Ap. J. (Letters), 164, L23.

Kerr, F. J., and Sandquist, A. 1968, Ap. Letters, 2, 195.

Penzias, A. A., Jefferts, K. B., and Wilson, R. W. 1971, Ap. J., 165, 229.

Penzias, A. A., Solomon, P. M., Wilson, R. W., and Jefferts, K. B. 1972, Ap. J. (Letters) (to be published).

Scoville, N. Z. 1972, Ap. J. (Letters), 175, 127.

Scoville, N. Z., Solomon, P. M., and Thaddeus, P. 1972, Ap. J., 172, 335.

Solomon, P. M. 1972, Symposium on Interstellar Molecules, ed. M. A. Gordon (New York: John Wiley \& Sons) (in press).

Solomon, P. M., Jefferts, K. B., Penzias, A. A., and Wilson, R. W. 1971, Ap. J. (Letters), 168, L107. 